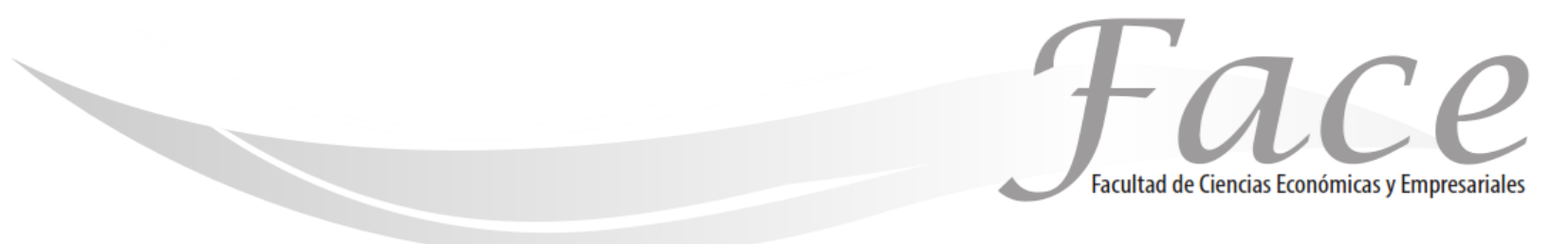

ISSN Impreso: 1794-9920 ISSN Electrónico: 2500-9338

Volumen $20-\mathrm{N}^{\circ} 2$

Año 2020

Págs. $107-119$

(c) (i) 8 (O)

\title{
LA LIBERTAD ECONÓMICA Y EL DESARROLLO ECONÓMICO-SOCIAL EN MÉXICO
}

\author{
Carmen Denisse Martínez Jiménez* \\ ORCID: https://orcid.org/0000-0002-0293-2966 \\ Elías Gaona Rivera** \\ ORCID: https://orcid.org/0000-0003-2630-219X
}

Fecha de Recepción: Agosto 30 de 2020

Fecha de Aprobación: Noviembre 29 de 2020

\section{Resumen:}

Existe evidencia fundamentada de diversos autores, además de la comparación entre países, que la libertad económica es esencial para el crecimiento y desarrollo económico de una nación, Por ello, el objetivo principal de esta investigación es analizar el desarrollo económico-social de 4 países, 3 que han adoptado medidas de libre mercado y con un sistema económico capitalista: EEUU, Corea del Sur; uno de marcada intervención estatal: Argentina; en comparación con México. Mediante el Índice de Libertad Económica y su evolución en México, se analizará si realmente por ello ha existido crecimiento económico; aunado a una revisión para determinar si dentro del régimen actual (democrático con un sistema de gobierno presidencial y una ideología "capitalista") es eficiente para las problemáticas enfrentadas día con día, como los altos niveles de pobreza y poca preparación escolar. Con ayuda de 3 escuelas de pensamiento económico: clásica, francesa y austriaca. Con los resultados analizados, se puede considerar que México tiende a una mayor libertad económica, aunque resulta sesgado, pues dentro de los parámetros (tamaño del gobierno, imperio de la ley, eficiencia regulatoria y mercados abiertos), algunos resultados pueden parecer importantes (llegando a ser considerados como fundamentales para que exista esta libertad), pero realmente no afecta en el resultado final, concluyendo que a pesar de que tienda a una mayor libertad, el desarrollo económico no es paritario, pues está concentrado para la élite gobernante en su mayoría, debido a la no creación de las mismas condiciones en cuanto a oportunidades.

Palabras clave: Libertad económica, crecimiento, ideología

Clasificación JEL: F41, F43, P51,

\footnotetext{
*Alumna de noveno semestre de la Licenciatura en Economía. Instituto de Ciencias Económico Administrativas. Universidad Autónoma del Estado de Hidalgo (UAEH), México . Contacto: cdenissemtz@outlook.es

* *Doctor Elías Gaona Rivera. Profesor Investigador en el Instituto de Ciencias Económico Administrativas. Universidad Autónoma del Estado de Hidalgo

(UAEH), México.Contacto: elias_gaona3473@uaeh.edu.mx
} 


\title{
ECONOMIC FREEDOM AND SOCIO-ECONOMIC DEVELOPMENT IN MEXICO
}

\begin{abstract}
:
There is well-founded evidence from various authors, in addition to the comparison between countries, that economic freedom is essential for the economic growth and development of a nation. Therefore, the main objective of this research is to analyze the socio-economic development of 4 countries that they have adopted free market measures, two of them with a capitalist economic system: the US and South Korea; and one of marked state intervention: Argentina; compared to Mexico. Through the Index of Economic Freedom and its evolution in Mexico, and analyze whether there has really been economic growth for this reason; coupled with a review to determine whether within the current regime (democratic with a presidential system of government and a "capitalist" ideology) it is efficient for the problems faced every day, such as high levels of poverty and poor school readiness. With the help of 3 schools of economic thought: classical, French and Austrian. With the results analyzed, it can be considered that Mexico tends towards greater economic freedom, although it is biased, since within the parameters (size of government, rule of law, regulatory efficiency and open markets), some results may seem important (reaching to be considered as fundamental for this freedom to exist), but it does not really affect the final result, concluding that although it tends to greater freedom, economic development is not equal, since it is concentrated for the majority ruling elite, due to not creating the same conditions in terms of opportunities.
\end{abstract}

Keywords: Economic freedom, growth, ideology

\section{LIBERDADE ECONÔMICA E DESENVOLVIMENTO}

\section{ECONÔMICO-SOCIAL NO MÉXICO}

\begin{abstract}
Resumo:
Existem evidências fundamentadas de diversos autores, além da comparação entre países, de que a liberdade econômica é essencial para o crescimento e desenvolvimento econômico de uma nação, portanto, o objetivo principal desta pesquisa é analisar o desenvolvimento econômico-social de 4 países, 3 que adotaram medidas de livre mercado e com sistema econômico capitalista: EUA, Coréia do Sul; um de marcada intervenção estatal: Argentina; em comparação com o México. Por meio do Índice de Liberdade Econômica e sua evolução no México, será analisado se realmente houve crescimento econômico; juntamente com uma revisão para determinar se dentro do regime atual (democrático com um sistema de governo presidencialista e uma ideologia "capitalista") é eficiente para os problemas enfrentados todos os dias, como altos níveis de pobreza e baixa escolaridade. Com a ajuda de 3 escolas de pensamento econômico: clássica, francesa e austríaca. Com os resultados analisados, pode-se considerar que o México tende a uma maior liberdade econômica, embora seja enviesado, pois dentro dos parâmetros (tamanho do governo, estado de direito, eficiência regulatória e mercados abertos), alguns resultados podem parecer importantes (atingindo ser considerada fundamental para que essa liberdade exista), mas não afeta realmente o resultado final, concluindo que embora tenda a uma maior liberdade, o desenvolvimento econômico não é igualitário, pois está concentrado para a elite dominante, pela não criação das mesmas condições em termos de oportunidades.
\end{abstract}

Palavras-chave: Liberdade econômica, crescimento, ideologia 


\section{MARCO TEORICO}

La libertad económica brinda a todos las mismas oportunidades para lograr la estabilidad financiera y la autonomía de propiedad, habilidades e ideas. También reduce la pobreza y mejora nuestra felicidad y el futuro de nuestra familia. Las investigaciones muestran que las personas que viven en países económicamente libres disfrutan de una mayor seguridad económica y es por ello que quizá muchos hispanos abandonan su país de origen en busca de una vida mejor en los Estados Unidos, por ejemplo. El principio de libertad económica ha demostrado ser la clave de su éxito a largo plazo.

Por lo tanto, el objetivo principal de este trabajo es analizar el progreso económico y social de tres países que han adoptado medidas de libre mercado y uno que va encaminado a ideales socialistas; Estados Unidos y Corea del Sur, que poseen un sistema de economía mixta que más se acerca al sistema económico capitalista; Argentina con una intervención estatal muy alta y México, que es propiamente capitalista y mantiene una economía de mercado pero con un enfoque social. Lo anterior, tomando en cuenta el análisis de 4 variables: tamaño del gobierno, imperio de la ley, eficiencia regulatoria y mercados abiertos, y de esta manera analizar si por separado son realmente significativas o si por el contrario, cuentan con un sesgo de medición por sí mismas.

Dentro del propósito a lograr con este investigación, es analizar qué tan viable es la libertad económica en México, comparándolo con otros países que tienen mayor o menor libertad, explicando el por qué una mayor libertad puede beneficiar y con ello lograr mayor plenitud en la población, aumentando su crecimiento y desarrollo económico, dando como resultado una exuberancia económica.

Desde que apareció el libro de Adam Smith, en 1776, la Riqueza de las Naciones existe un debate entre qué sistema económico es el mejor para el progreso de los países: 1) El que tiene una amplia participación del estado en la economía y 2) en donde el estado pretende estar ausente o tiene una participación mínima. De acuerdo con Adam Smith el mercado libre actúa como una "mano invisible" que maximiza el bienestar general. Ningún país en el mundo se gobierna totalmente de acuerdo con los ideales libertarios y ningún país es completamente socialista, pero existen grandes diferencias entre las naciones de acuerdo a su grado de capitalismo y socialismo. $Y$ sin duda alguna para los economistas siempre ha sido un tema fundamental de discusión, puesto que a partir de ahí es como un país puede o no prosperar.
Se pueden encontrar múltiples trabajos, tesis, libros, etc. en donde los autores nos demuestran que a mayor libertad económica y menor intervención del estado, se logra un mayor progreso económico-social que da pauta a un mayor crecimiento y desarrollo económico de las naciones y que por lo tanto se eleva el nivel de bienestar de la población.

Las corrientes de pensamiento económico tomadas para la elaboración de esta investigación fueron 3 , las cuales van encaminadas hacia la defensa de las libertades individuales en el ámbito económico.

Dentro de la economía clásica encontramos a Adam Smith con su famosa obra "Teoría de los Sentimientos Morales", publicada en 1759, y en la que menciona el término de la famosa "mano invisible" por primera vez, pero es hasta 1776 que tomó más fuerza en otro libro titulado "La riqueza de las naciones", en donde es presentada una metáfora económica que intentaba demostrar la existencia de una ley de la naturaleza, la cual funcionaría con más eficacia cuanto menos se perturbara, es decir, en un mercado libre, y que el efecto combinado de que todas las personas busquen su interés propio, beneficia a la sociedad en conjunto directa 0 indirectamente y que incluso la toma de decisiones individuales puede ser más apropiada que el mismo gobierno. Sostiene que la libre competencia es la mejor manera de hacer funcionar a la economía y que esta mano invisible tendría la capacidad de autorregular al mercado, además supone que se estimula o se frena a los individuos a producir o no siguiendo el nivel de precios que existen en el mercado en base a la oferta y la demanda. $Y$ que por ello es que el gobierno debe limitarse a la defensa o justicia, dejando al mercado a sus libre funcionamiento. Aunque resalta que en algunas situaciones esta teoría no era apropiada.

En segundo término se estudió a Frederic Bastiat, que pertenece a la escuela liberal francesa y desarrolló un pensamiento liberal e individualista, caracterizado por la defensa del libre comercio y la oposición al socialismo y al colonialismo y que defiende la libertad del individuo frente a toda autoridad, lo cual está plasmado en dos de sus ensayos muy famosos, "La Ley" (1849) y "El estado"(1848). En el primero habla de que el individuo es nuestra razón de ser, y que toda forma de Estado 
debe beneficiar al individuo, pero éste va perdiendo su sentido cuando se gira en contra de él, haciendo referencia a que la ley se pervierte y en lugar de usarse para esa defensa se usa para atacar aún más al individuo. Aquí menciona que la ley debe defender los 3 principios: persona, libertad y propiedad. En el segundo ensayo "El Estado", da argumentos en contra del socialismo, y menciona tal vez su cita más conocida: "El Estado es la gran ficción mediante la cual todo el mundo trata de vivir a expensas de los demás", haciendo referencia por ejemplo al pago de los impuestos. A veces la sociedad prefiere aceptar mayores impuestos a cambio de mantener el "Estado de Bienestar", en lugar de menores impuestos y una mayor participación de los privados, y esto precisamente haciendo real otra frase de Bastiat: "prevalece el despojo siempre que sea menos costoso que el trabajo". Y en este trabajo concluye que los pueblos más civilizados son aquellos en los que el estado interviene menos.

Por último, se analizó a la escuela austriaca quien es quizá la corriente en la que se puede notar un mayor apoyo a la libertad económica, incluso tiende a autodefinirse como «la ciencia económica del libre mercado, a pesar de las tantas críticas que se le hacen por la metodología que utiliza, pues la mayoría de los autores rechazan la metodología de otras escuelas de pensamiento económico como el keynesianismo o el monetarismo, pues adoptaron métodos empíricos, matemáticos y estadísticos y se centraron en la inducción para construir y probar teorías, al contrario de la escuela austriaca que dice que la ciencia económica se deriva de la lógica filosófica, y las teorías económicas razonables solo pueden desarrollarse a partir de principios lógicos básicos, pero lo que interesa analizar en este caso es a las conclusiones que llegan dos autores destacados de esta escuela en sus trabajos.

Hayek (1960) en su libro titulado "Los fundamentos de la libertad", nos da un panorama y nos dice que los principios fundamentales de la libertad en realidad son las condiciones para lograr la riqueza y el crecimiento, y no al contrario. Este trabajo se considera en general como una de las expresiones más importantes del pensamiento político del siglo XX. Dedicado a analizar la "constitución" o estructura de la libertad y su significado en la sociedad contemporánea, este es el resultado de una cuidadosa investigación en los campos de la filosofía política, el derecho y la economía. Se divide principalmente en tres partes. En la primera, mostró por qué queremos la libertad y qué trae la libertad. Esta es una importante discusión teórica y filosófica que involucra el estudio de los factores que determinan el progreso de la civilización. La segunda parte es una investigación del sistema establecido por Occidente para asegurar la libertad individual y resolver estos problemas desde una perspectiva histórica, con miras a promover la solución de los problemas actuales basada en ideales sólo parcialmente realizados. La tercera parte es la aplicación práctica en algunas de las situaciones económicas y sociales clave de la actualidad y, lo más importante, se centra en los problemas en los que la elección incorrecta entre las posibles soluciones causa el mayor daño a la libertad.

Ludwig Von Mises(1922) también fue un gran defensor del movimiento liberal-libertario, pues estaba a favor del libre mercado y decía que la intervención gubernamental en la economía no era natural y que por lo tanto generaba caos a largo plazo, perjudicando a la sociedad. Y fue en su obra EL SOCIALISMO; Análisis económico y sociológico donde afirmó que el sistema comunista carecía de un mecanismo de precios y por lo tanto, la asignación de recursos era ineficiente, al contrario del sistema capitalista y que por lo tanto este sistema llevaba inevitablemente al fracaso y se debían evitar valoraciones éticas y morales.

Siempre ha existido un "debate" respecto a las ideologías políticas y económicas, sobre qué forma de gobierno y qué sistema económico es el mejor para tener un país más próspero, ambas van muy de la mano, incluso me atrevería a decir que no existe una sin la otra, al menos en la realidad en la que vivimos. Ambas van dirigidas a saber cómo es que debería funcionar la sociedad en conjunto.

El capitalismo mexicano se desarrolla en los marcos del proceso de conformación del capitalismo como una economía global en el siglo XIX... La consolidación del capitalismo en nuestro país se da cuando la expansión en los flujos internacionales de materias primas, manufacturas, capitales y fuerza de trabajo, alcanzan dimensiones sin precedentes, bajo el impulso productivo de la segunda revolución industrial (Villalobos, 2012: 27)

México ha tenido desde hace años un régimen democrático, un sistema de gobierno presidencial y una ideología "capitalista"; entendiendo esta como un sistema económico y social, el cual se basa en la propiedad privada de los medios de producción, en la importancia del capital como generador de riqueza y en la asignación de los recursos a través del mecanismo del mercado, aunque a partir de que comenzó el sexenio del actual presidente, Andrés Manuel López Obrador, todo lo anteriormente expuesto ha llevado a poner en duda nuestro sistema, pues se han implementado una serie 
de cambios, dentro de los cuales se puede notar un enfoque social, encaminado a las personas que se encuentran en situación de pobreza, entendida como la escasez de bienes materiales necesarios para poder subsistir y la parte empresarial y de mercado causa incertidumbre porque de alguna manera se está dejando de lado, atravesamos una coyuntura importante.

Ahora bien, Cuando hablamos de LIBERTAD ECONÓMICA, Katz I. (2019) resalta los siguientes elementos:

a) los derechos privados de propiedad están eficientemente definidos en el marco legal;

b) los individuos tienen la libertad irrestricta de poseer bienes y recursos productivos;

c) los agentes económicos son libres de utilizar los recursos de su propiedad, incluido su propio cuerpo, para cualquier fin mientras en el ejercicio de tal libertad no se atente en contra de los derechos de terceros;

d) los agentes económicos son libres para transferir los recursos de su propiedad en transacciones enteramente voluntarias, tanto nacional como internacionalmente;

e) los derechos de propiedad así como las tres libertades señaladas son protegidas y garantizadas por un Poder Judicial independiente e imparcial que además garantiza el cumplimiento de los contratos.

Dentro del gran debate al principio mencionado, está el que a todos nos hace estar en dos "bandos" diferentes, pues existe evidencia de que entre mayor grado de libertad económica, mayores beneficios existen para la sociedad que comprende ese núcleo, existe mayor desarrollo y crecimiento económico para los países que así se rigen, por lo tanto, todos salen ganando.

\section{METODOLOGÍA}

La metodología utilizada fue la técnica del índice de libertad económica dentro del periodo de 1995 a 2020, haciendo una comparación entre los cuatro países ya antes mencionados. Dicho índice fue creado y es publicado por la Fundación Heritage en conjunto con el Wall Street Journal.

La hipótesis es que los países con mayor progreso económico-social son los que han adoptado medidas capitalistas más liberales, basadas en el libre mercado con la poca intervención del estado.

El índice en el que se basa principalmente este artículo es el Índice de Libertad Económica, publicado por The Heritage Foundation; el cual tiene cuatro grandes agregados, y estos a su vez tienen distintos rubros ( 3 cada uno) que definen si una nación es más libre económicamente, en donde 100 significa "libertad económica total" y 0 "sin libertad económica" los cuales siguientes son:

$\checkmark$ Tamaño del gobierno: Carga fiscal, Gastos gubernamentales y Salud fiscal

$\checkmark$ Imperio de la ley: Derechos de Propiedad, Efectividad Judicial e Integridad del Gobierno

$\checkmark$ Eficiencia Regulatoria: libertad comercial, libertad laboral y libertad monetaria

$\checkmark$ Mercados abiertos: libertad de comercio, libertad de inversión y libertad financiera

Además, dentro de la información recabada se hace un análisis de la tasa arancelaria, tasa de impuesto sobre la renta, tasa de impuesto comparativa, la carga fiscal del $\mathrm{PIB}$, gasto del gobierno del PIB, su población, PIB a PPA, tasa de crecimiento del PIB, tasa de crecimiento del PIB a 5 años, PIB per cápita, desempleo, inflación, entrada de inversión extranjera y la deuda publica en un periodo de 2013-2020 


\section{RESULTADOS}

En la siguiente tabla se muestran los países que se analizarán y la puntuación dentro del ranking para el año 2020:

Tabla 1.

Ranking de libertad económica 2020

\begin{tabular}{c|c|c}
\hline Rango & País & Puntuación \\
\hline 17 & Estados Unidos & 76.6 \\
\hline 25 & Corea del Sur & 74.0 \\
\hline 67 & México & 66.0 \\
\hline 149 & Argentina & 53.1 \\
\hline
\end{tabular}

Fuente: elaboración propia con datos de: Índice de Libertad Económica, The Heritage Foundation, 2020

En la siguiente tabla se muestran los periodos en que cada presidente estuvo al mando del país y el puntaje correspondiente, obteniendo así un promedio por sexenio y uno en general del periodo de 1995 a 2020

Tabla 2.

Índice de grado de libertad económica de México 19952020.

\begin{tabular}{|c|c|c|c|}
\hline Sexenio & $\begin{array}{l}\text { Año } \\
\text { índice }\end{array}$ & $\begin{array}{l}\text { Puntaje } \\
\text { general }\end{array}$ & $\begin{array}{c}\text { Promed } \\
\text { io por } \\
\text { sexenio }\end{array}$ \\
\hline \multirow{2}{*}{$\begin{array}{c}\text { Andrés Manuel } \\
\text { López Obrador } \\
\text { (2018-2014) }\end{array}$} & 2020 & 66 & \multirow[t]{2}{*}{65.35} \\
\hline & 2019 & 64.7 & \\
\hline \multirow{9}{*}{$\begin{array}{c}\text { Enrique Peña } \\
\text { Nieto (2012-2018) }\end{array}$} & 2018 & 64.8 & \multirow[t]{6}{*}{65.58} \\
\hline & 2017 & 63.6 & \\
\hline & 2016 & 65.2 & \\
\hline & 2015 & 66.4 & \\
\hline & 2014 & 66.8 & \\
\hline & 2013 & 67 & \\
\hline & 2012 & 65.3 & \multirow[t]{3}{*}{66.3} \\
\hline & 2011 & 67.8 & \\
\hline & 2010 & 68.3 & \\
\hline
\end{tabular}

\begin{tabular}{|c|c|c|c|}
\hline \multirow{3}{*}{$\begin{array}{c}\text { Felipe Calderón } \\
\text { Hinojosa } \\
(2006-2012)\end{array}$} & 2009 & 65.8 & \\
\hline & 2008 & 66.2 & \\
\hline & 2007 & 66 & \\
\hline \multirow{6}{*}{$\begin{array}{l}\text { Vicente Fox } \\
\text { Quesada } \\
(2000-2006)\end{array}$} & 2006 & 64.7 & \multirow[t]{6}{*}{64.13} \\
\hline & 2005 & 65.2 & \\
\hline & 2004 & 66 & \\
\hline & 2003 & 65.3 & \\
\hline & 2002 & 63 & \\
\hline & 2001 & 60.6 & \\
\hline \multirow{7}{*}{$\begin{array}{c}\text { Ernesto Zedillo } \\
\text { Ponce de León } \\
\text { (1994-2000) }\end{array}$} & 2000 & 59.3 & \multirow[t]{6}{*}{59.51} \\
\hline & 1999 & 58.5 & \\
\hline & 1998 & 57.9 & \\
\hline & 1997 & 57.1 & \\
\hline & 1996 & 61.2 & \\
\hline & 1995 & 63.1 & \\
\hline & $\begin{array}{c}\text { PROMED } \\
10\end{array}$ & $\begin{array}{c}64.069230 \\
77\end{array}$ & \\
\hline
\end{tabular}

Fuente: elaboración propia con datos de: Índice de Libertad Económica, The Heritage Foundation, 2020

Teniendo el sexenio con mayor libertad económica bajo las políticas aplicadas en el mismo, el de Felipe Calderón Hinojosa en el periodo de 2006 a 2012

A su vez, la siguiente gráfica muestra una comparación de México con del resto del mundo, teniendo una tendencia a ser moderadamente libre.

\section{Gráfica 1.}

\section{Puntuación de libertad económica 1995-2020: México y el mundo.}

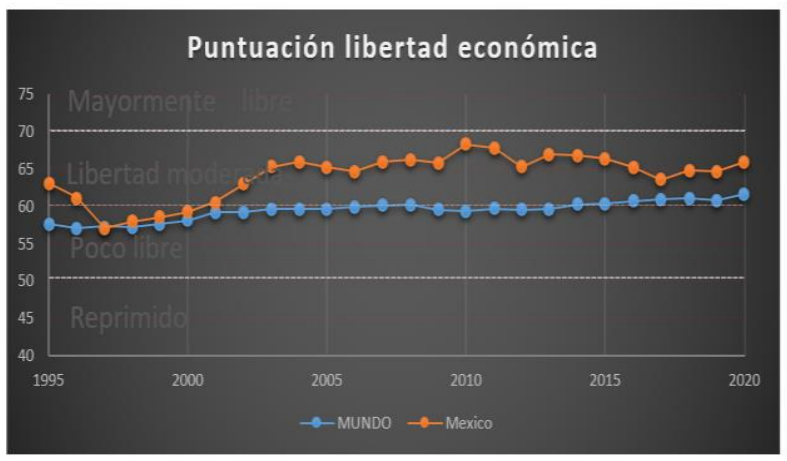

Fuente: elaboración propia con datos de: Índice de Libertad Económica, The Heritage Foundation, 2020 
La economía mexicana ha sido clasificada moderadamente libre durante prácticamente todos los años analizados. El crecimiento moderado del PIB en los últimos cinco años comenzó a desacelerarse en 2018, lo que refleja la débil confianza de los inversores en medio de la incertidumbre política de la nueva administración, las finanzas inestables de la compañía petrolera estatal Pemex y las preguntas sobre las futuras relaciones comerciales con los EE. UU.

La corrupción sigue siendo un problema grave en México. Si bien el indicador de integridad del gobierno mejoró en 2020, quizás en parte como resultado de los esfuerzos anticorrupción del presidente, México aún se encuentra en la mitad inferior del Índice en esta área.

Es necesario recordar que el Partido Revolucionario Institucional (PRI) gobernó durante 70 años seguidos, hasta que en el año 2000 el Partido Acción Nacional (PAN) ganó las elecciones con Felipe Calderón Hinojosa, pero en 2012 de nuevo el PRI recuperó la presidencia con Enrique Peña Nieto. Pero en el año 2018 Andrés Manuel López Obrador, partidista de MORENA obtuvo el puesto presidencial, teniendo como plan la "4T" (cuarta transformación), en la cual abarca medidas anticorrupción y políticas nacionalistas.

En 2018 se llevaron a cabo las elecciones federales de México, que como ya se mencionó anteriormente, el partido Movimiento Regeneración Nacional (Morena) fue el ganador $y$ es quien ha hecho que alrededor de 30 millones de mexicanos (quienes votaron por dicha opción) mantengan la ilusión de un verdadero cambio.

Si bien es cierto, cada proceso electoral despierta nuevas esperanzas en los habitantes de cada país, Andrés Manuel López Obrador, ha representado un acrecentamiento del sistema democrático, además del proceso de alternancia política que desde el inicios del siglo XX ha ido teniendo notoriedad. El triunfo de López Obrador significa tener a un candidato procedente de un partido de izquierda al frente de la presidencia del país por primera vez; vale la pena señalar que la estrategia de campaña del candidato ganador siempre se ha basado en una fuerte crítica a las políticas del gobierno en las últimas décadas y en la promesa de un cambio real. En definitiva, el nuevo gobierno democrático trascendió el hecho de que ganó la victoria democrática 0 candidato de izquierda, e hizo su propuesta básica para terminar con 36 años de neoliberalismo y comenzar un plan de transformación del país.

Si bien, el neoliberalismo tiene un principio ideológico político, es una respuesta a las condiciones opresivas del sistema feudal impuestas por los reyes de las monarquías absolutistas en la Europa del siglo XVIII (Fernández, 2003): (Boaz, 2007), el neoliberalismo es un modelo económico dominante de finales del siglo XX y principios del XXI, tiene su origen a partir de los trabajos de Herbert Spencer, Jacques Rueff y Friedrich Hayek, todos ellos fundamentados en la obra seminal An Inquiry into the Nature and Causes of the Wealth of Nations escrita por Adam Smith y publicada en 1776 (Cardoso, 2006).

La ortodoxia de la política económica mexicana desde fines de la década de 1980 ha sido descrita en la literatura como neoliberal. Si bien se basa principalmente en la aplicación de la privatización, la liberalización comercial y financiera y la desregulación de reformas económicas, tiene varios elementos para reducir la participación del Estado en la economía y permitir que los precios distribuyan el libre poder de mercado. Además de esto, también se busca la estabilidad macroeconómica, principalmente para controlar la inflación (baja inflación).

En todas las situaciones anteriores, no se tiene en cuenta el objetivo del crecimiento económico y el empleo, y mucho menos el bienestar, la equidad y el desarrollo económico. Existe una gran cantidad de literatura que analiza y discute los beneficios, pérdidas, ventajas y desventajas del neoliberalismo. Para el economista Ha-Joon Chang, luego de que el gobierno implementó el paradigma neoliberal durante 36 años, la economía mexicana ha mostrado un enorme e importante déficit, especialmente en términos de crecimiento económico, política industrial y equidad (Chang, 2019)

En las siguientes tablas se hace un concentrado de promedios para cada país, en donde se ve reflejada la ponderación general dentro del periodo de 1995 a 2020 en cada variable tomada para su medición. 
Tabla 3.

Tamaño del gobierno.

\begin{tabular}{l|c|c|c}
\hline & Carga fiscal & $\begin{array}{c}\text { Gastos } \\
\text { gubernamentales }\end{array}$ & $\begin{array}{c}\text { Salud } \\
\text { fiscal }\end{array}$ \\
\hline $\begin{array}{l}\text { Estados } \\
\text { Unidos }\end{array}$ & 66.87 & 58.35 & 53.87 \\
\hline $\begin{array}{l}\text { Corea del } \\
\text { Sur }\end{array}$ & 70.55 & 76.76 & 96.97 \\
\hline México & 76.26 & 82.58 & 76.82 \\
\hline Argentina & 71.04 & 70.65 & 41.67 \\
\hline
\end{tabular}

Fuente: elaboración propia con datos de: Índice de Libertad Económica, The Heritage Foundation, 2020

El primer rubro evaluado es el tamaño de gobierno, el cual engloba 3 variables más:

1.- Carga fiscal: en donde se analizan las tasas tributarias sobre el ingreso personal y corporativo y el nivel general de impuestos (directos e indirectos) como parte porcentual del PIB. México tiene en promedio 76.27 puntos, es decir, tiene en promedio una alta tasa impositiva, y en comparación con los otros países, entre más alto sea este valor, menos es el nivel del libertad en el mercado que se tiene. Las variables englobadas en este rubro son: tasa máxima de impuesto marginal sobre el ingreso individual, tasa máxima de impuesto marginal sobre el ingreso corporativo y carga fiscal total como porcentaje del PIB.

2.- Gastos gubernamentales: Calcula la carga del gasto público, incluyendo el consumo por parte del estado y todos los pagos de transferencia relacionados con varios programas de ayuda social. El nivel ideal varía de un país a otro, pero el gasto cero se utiliza como punto de referencia. México cuenta con 82.58 puntos en este rubro, lo que confirma lo anteriormente señalado en cuanto a sus políticas encaminadas al aspecto de ayudas sociales, lo que representa una posible relación con la alta recaudación de impuestos.

3.-Salud fiscal: Examina qué tan bueno es el manejo del presupuesto de un país, cuantificando la creciente deuda y déficit. $Y$ su cálculo incluye: el déficit promedio como porcentaje del PIB en los últimos tres años (80 por ciento del puntaje) y la deuda como porcentaje del 114
PIB (20 por ciento del puntaje). En este sentido, México tiene 76.82 puntos, interpretados como un alto nivel de deuda pública en relación al tamaño de su economía.

Tabla 4.

Imperio de la ley.

\begin{tabular}{c|c|c|c}
\hline & $\begin{array}{c}\text { Derechos } \\
\text { de } \\
\text { propiedad }\end{array}$ & $\begin{array}{c}\text { Efectivida } \\
\text { d judicial }\end{array}$ & $\begin{array}{c}\text { Integridad } \\
\text { del gobierno }\end{array}$ \\
\hline $\begin{array}{c}\text { Estados } \\
\text { Unidos }\end{array}$ & 86.60 & 78.57 & 76.02 \\
\hline $\begin{array}{c}\text { Corea del } \\
\text { Sur }\end{array}$ & 77.84 & 60.52 & 51.58 \\
\hline México & 52.08 & 36.82 & 34.17 \\
\hline Argentina & 38.90 & 43.9 & 34.48 \\
\hline
\end{tabular}

Fuente: elaboración propia con datos de: Índice de Libertad Económica, The Heritage Foundation, 2020

Dentro del imperio de la ley se encuentran 3 rubros:

1.-Derechos de propiedad, el cual hace referencia al grado de protección legal de los derechos de propiedad privada y el grado de cumplimiento de esas leyes. A su vez abarca: Derechos de propiedad física, de propiedad intelectual, la fuerza de protección del inversor, el riesgo de expropiación y la calidad de la administración de tierras; en este caso México tiene un puntaje de 52, mostrando la ineficiencia del derecho a la propiedad a pesar de lo estipulado en el Artículo 27 de la Constitución Política de los Estados Unidos Mexicanos.

2.- En cuanto a la efectividad judicial tiene un muy bajo puntaje, teniendo un promedio de 36.82 , esta variable engloba el grado de eficiencia e imparcialidad del poder judicial, especialmente en lo que respecta a las leyes de propiedad, dividiéndose a su vez en independencia judicial, calidad del proceso judicial y la probabilidad de obtener decisiones judiciales favorables.

3.- La integridad del gobierno examina en qué grado sobresalen las formas de corrupción política y prácticas como soborno, extorsión, nepotismo, amiguismo, mece nazgo y malversación de fondos. Englobando así la confianza pública en políticos, el pagos irregulares y sobornos, la transparencia de la formulación de 
políticas del gobierno, la ausencia de corrupción, las percepciones de corrupción y la transparencia gubernamental y del servicio civil. Teniendo como promedio un puntaje sumamente bajo 34.17, lo cual resulta muy lógico, pues siempre han sido criticados estos aspectos en la nación mexicana.

\section{Tabla 5.}

Eficiencia regulatoria.

\begin{tabular}{c|c|c|c}
\hline & $\begin{array}{c}\text { Libertad } \\
\text { comercial }\end{array}$ & $\begin{array}{c}\text { Libertad } \\
\text { laboral }\end{array}$ & $\begin{array}{c}\text { Libertad } \\
\text { monetaria }\end{array}$ \\
\hline $\begin{array}{c}\text { Estados } \\
\text { Unidos }\end{array}$ & 87.11 & 94.18 & 81.46 \\
\hline Corea del Sur & 81.60 & 52.8 & 81.26 \\
\hline México & 69.31 & 60.10 & 73.43 \\
\hline Argentina & 67.92 & 45.55 & 68.80 \\
\hline
\end{tabular}

Fuente: elaboración propia con datos de: Índice de Libertad Económica, The Heritage Foundation, 2020

Para el análisis de este rubro se engloban:

1.- La libertad comercial, que analiza el costo, el tiempo y la libertad de abrir, operar y cerrar un negocio, el cual a su vez toma en cuenta 13 factores como el número de procedimientos para iniciar un negocio, el tiempo (en días) de inicio de un negocio, el costo (\% ingreso per cápita) para iniciar un negocio, entre otros, teniendo un puntaje medio de 69.31, es decir, para las personas es más complicado iniciar un negocio en México por los costos y procedimientos que se requieren, que en otros países como en EEUU o Corea del Sur.

2.- El objetivo de la libertad laboral es cuantificar las violaciones de los derechos laborales, como los salarios mínimos, las leyes que prohíben los despidos, los requisitos de indemnización y las restricciones mensurables y ajustables sobre la contratación y las horas de trabajo, y las tasas de participación de la fuerza laboral como indicadores de oportunidades de empleo en el mercado laboral. Algunos de los factores en los que se divide son: la relación entre el salario mínimo y el valor agregado promedio por trabajador, el impedimento para contratar trabajadores adicionales, la tasa de participación en la fuerza laboral, entre otras. Los 60 puntos alcanzados por México nos indican que se requiere que las reformas laborales contemplen esta incertidumbre que viven las personas dentro del sector laboral.
3.- La libertad monetaria refleja qué tan estables son los precios y cuánto interviene la microeconomía. Se divide en el promedio ponderado de la tasa de inflación en los últimos tres años y en los controles de precios. México tiene un promedio de 73.43 , indicando que la nación necesita una moneda estable y confiable como medio para intercambiar y almacenar valor.

Tabla 6.

\section{Mercados abiertos.}

\begin{tabular}{c|c|c|c}
\hline & $\begin{array}{c}\text { Libertades } \\
\text { de comercio }\end{array}$ & $\begin{array}{c}\text { Libertad de } \\
\text { inversión }\end{array}$ & $\begin{array}{c}\text { Libertad } \\
\text { financiera }\end{array}$ \\
\hline $\begin{array}{c}\text { Estados } \\
\text { Unidos }\end{array}$ & 83.15 & 73.65 & 76.92 \\
\hline $\begin{array}{c}\text { Corea del } \\
\text { Sur }\end{array}$ & 71.91 & 67.5 & 63.46 \\
\hline México & 77.20 & 62.88 & 54.61 \\
\hline Argentina & 64.43 & 53.26 & 46.92 \\
\hline
\end{tabular}

Fuente: elaboración propia con datos de: Índice de Libertad Económica, The Heritage Foundation, 2020

En la última variable a analizar se engloban 3 parámetros:

1.- Libertades de comercio, que con 77.20 puntos, cuantifica la medida en qué aranceles y barreras no arancelarias afectan importaciones y exportaciones de bienes y servicios dentro y fuera del país, arrojando como resultado una fortaleza de la nación, pues de hecho es quien más acuerdos y tratados comerciales tiene con el resto del mundo.

2.-La libertad de inversión cuantifica que tan libre o limitado es el flujo de capital de inversión de individuos y empresas, teniendo como resultado 62.88 puntos promedio, pues en México la inversión extranjera directa ha crecido a lo largo de los años.

3.-La libertad financiera analiza la eficiencia del banco así como cuán independiente es el gobierno del sector financiero. Este aspecto analiza cinco áreas, algunas son: extensión de la regulación gubernamental de los servicios financieros, grado de intervención estatal en bancos y otras empresas financieras a través de la propiedad directa e indirecta y la influencia del gobierno en la asignación de crédito, obteniendo así un promedio de 54.61 puntos a lo largo de los años, lo que significa que se requiere de un ambiente más favorable 
para hacer negocios para la obtención de una economía más dinámica.

Tabla 7.

Puntaje general.

\begin{tabular}{c|c}
\hline $\begin{array}{c}\text { Estados } \\
\text { Unidos }\end{array}$ & 77.46 \\
\hline Corea del Sur & 70.35 \\
\hline México & 64.06 \\
\hline Argentina & 56.75
\end{tabular}

Fuente: elaboración propia con datos de: Índice de Libertad Económica, The Heritage Foundation, 2020

Desde 1995 hasta hoy en día, México se ha mantenido relativamente estable en cuando a su libertad económica, teniendo un puntaje promedio a lo largo de este período de 64 puntos, es decir, cuenta con una libertad económica moderada y con amplias oportunidades de crecimiento y desarrollo con la apertura de espacios libres para el buen funcionamiento del mercado.

Dentro de los rubros que se miden en este índice para México, se describe en general de la siguiente manera:

\section{Tamaño del gobierno:}

La tasa impositiva máxima sobre los ingresos individuales es del $35 \%$, y la tasa impositiva corporativa es del $30 \%$. Otros impuestos incluyen un impuesto al valor agregado. La carga impositiva general equivale al $16,2 \%$ del ingreso interno total. El gasto gubernamental ha ascendido al $26,3 \%$ de la producción (PIB) del país en los últimos tres años, y los déficits presupuestarios han promediado el 2,1 por ciento del PIB. La deuda pública es equivalente al $53,6 \%$ del PIB.

\section{Imperio de la ley:}

Los derechos de propiedad están protegidos por la ley, pero un poder judicial débil, la frecuente solicitud de sobornos por parte de burócratas y funcionarios, y la alta incidencia de extorsión criminal socavan la seguridad de la propiedad. El

sistema de justicia de México está plagado de demoras, imprevisibilidad y corrupción, lo que lleva a la impunidad. Una de las principales prioridades declaradas por el presidente López Obrador es combatir la corrupción, que es generalizada y alimentada por miles de millones de narcodólares.

\section{Eficiencia regulatoria:}

Los aumentos en algunas tarifas que afectan los permisos de construcción han afectado el entorno empresarial. La incertidumbre sobre la ejecución del contrato y la inseguridad física son preocupaciones entre los propietarios de negocios. Más personas trabajan de manera informal de lo habitual en un país con un PIB per cápita tan alto como el de México. La reversión de reformas previas por parte del nuevo gobierno ha aumentado considerablemente el costo de los subsidios y el drenaje presupuestario de empresas estatales ineficientes.

\section{Mercados abiertos:}

El valor total de las exportaciones e importaciones de bienes y servicios equivale al 80,3 por ciento del PIB. La tasa arancelaria promedio aplicada es de 1.2 por ciento, y están vigentes 240 medidas no arancelarias. A pesar del fuerte deseo de atraer más inversión extranjera, el régimen de inversión carece de eficiencia y se ve obstaculizado por la inestabilidad. El sector financiero se ha vuelto más competitivo y abierto, y el sistema bancario sigue relativamente bien capitalizado.

Para complementar la investigación, se presentan algunos datos englobados dentro de los subfactores de las variables tomadas para poder entender mejor el contexto de los resultados arrojados anteriormente y para ello se obtuvo un promedio desde el año 2013 hasta el 2020.

Tabla 8.

Indicadores macroeconómicos.

\begin{tabular}{l|c|c|c|c}
\hline & $\begin{array}{c}\text { Tasa } \\
\text { arancelaria } \\
(\%)\end{array}$ & $\begin{array}{c}\text { Tasa de } \\
\text { impuesto } \\
\text { sobre la } \\
\text { renta } \\
(\%)\end{array}$ & $\begin{array}{c}\text { Tasa de } \\
\text { impuesto } \\
\text { corporativa } \\
(\%)\end{array}$ & $\begin{array}{c}\text { Carga } \\
\text { fiscal\% } \\
\text { del } \\
\text { PIB }\end{array}$ \\
\hline $\begin{array}{l}\text { Estados } \\
\text { Unidos }\end{array}$ & 1.715 & 38.37 & 31.5 & 25.64 \\
\hline $\begin{array}{l}\text { Corea del } \\
\text { Sur }\end{array}$ & 6.86 & 39.27 & 24.43 & 25.64 \\
\hline México & 2.93 & 33.75 & 30 & 15.05 \\
\hline Argentina & 6.62 & 35 & 33.75 & 32.06 \\
\hline
\end{tabular}


diferentes. Si uno separa las economías del mundo en

\begin{tabular}{l|l|l|l}
\hline & $\begin{array}{l}\text { Gasto del } \\
\text { gobierno\% } \\
\text { del PIB }\end{array}$ & $\begin{array}{l}\text { Población } \\
\text { (millones) }\end{array}$ & $\begin{array}{l}\text { PIB } \\
\text { (miles de } \\
\text { millones, } \\
\text { PPA) }\end{array}$ \\
\hline $\begin{array}{l}\text { Estados } \\
\text { Unidos }\end{array}$ & 39.25 & 319.91 & 17674.76 \\
\hline Corea del Sur & 31.89 & 50.58 & 1820.18 \\
\hline México & 26.96 & 119.79 & 2121.84 \\
\hline Argentina & 41.17 & 42.50 & 858.30 \\
\hline
\end{tabular}
cuatro grupos por su nivel de libertad, encuentra que el ingreso per cápita de los más libres es de US $\$ 41.000$, mientras que el próximo grupo goza de la mitad de ese ingreso.

Quienes defienden la libertad económica defienden una forma de organizar la actividad económica en la sociedad que beneficia a todos, o dicho de otra manera, que está orientado a satisfacer las necesidades y deseos del consumidor. $Y$ consumidores somos todos. (Placer J.L., 2018)

La libertad económica es la que ha hecho posible el consumo universal, a lo que hoy llamamos Globalización,(término utilizado con más frecuencia a partir de los 80's en el siglo XX, a pesar de que su origen y desarrollo lleva mucho más tiempo), el cual es un proceso en donde interactúan y se integran las personas (empresas y gobiernos) de diferentes países. Dicha interacción engloba lo económico, tecnológico político, social y cultural, es decir, ha crecido la comunicación e interdependencia. Este concepto engloba propuestas teóricas que abarca dos grandes tendencias: los sistemas de comunicación mundial y las condiciones económicas. La globalización es llegada a considerar como la modernización del modelo tradicional de producción capitalista

La característica fundamental del ser humano es su capacidad creativa y esta solo puede desarrollarse plenamente en un marco de libertad económica.

\begin{tabular}{l|l|l|l|l}
\hline & $\begin{array}{l}\text { Desempleo } \\
(\%)\end{array}$ & $\begin{array}{l}\text { Inflación } \\
(\%)\end{array}$ & $\begin{array}{l}\text { Entrada de } \\
\text { IED } \\
\text { (millones) }\end{array}$ & $\begin{array}{l}\text { Deuda } \\
\text { pública } \\
(\% \text { del } \\
\text { PIB) }\end{array}$ \\
\hline $\begin{array}{l}\text { Estados } \\
\text { Unidos }\end{array}$ & 6.02 & 1.77 & 246584.37 & 105.68 \\
\hline $\begin{array}{l}\text { Corea } \\
\text { del Sur }\end{array}$ & 3.53 & 1.72 & 10510.6 & 36.89 \\
\hline México & 4.37 & 3.97 & 26452.087 & 50.46 \\
\hline Argentina & 7.66 & 26.61 & 9613.39 & 53.90 \\
\hline
\end{tabular}

Fuente: elaboración con datos de: Índice de Libertad Económica, The Heritage Foundation, 2020.

Para Vásquez I. (2016) no es casualidad que la evidencia mundial muestra que a más libertad económica, mayor prosperidad y mayor crecimiento. El aumento de libertad incrementa tanto la productividad como el nivel y la calidad de las inversiones, lo cual conlleva a más riqueza. Lo impresionante es que aumentos aparentemente menores de libertad económica producen resultados dramáticamente

Por su puesto siempre habrán cosas buenas y cosas malas de cada idea que se tenga, el punto fundamental es saber aplicar realmente los conceptos a la práctica, de manera que exista la menor afectación posible, siempre sabiéndose adaptar a los cambios que esto conlleve, pues al final el resultado será con fines de bienestar, crecimiento y desarrollo para todos. 


\section{CONCLUSIONES:}

En términos generales se observa una alta correlación entre el grado de libertad económica y el buen desempeño de la economía de los países

Después de analizar los datos recabados, se puede concluir que en general y bajo los parámetros marcados, México es un país con una libertad económica moderada, con una tendencia a ser mayormente libre; aunque se concuerda con los sesgos mencionados anteriormente, pues por algunas puntuaciones elevadas como lo es dentro de la apertura al comercio, las que tienen un puntaje más bajo y que se podría considerar son fundamentales para tener una mayor libertad económica individual, es dentro del imperio de la ley. A pesar de que parece ser que no genera un gran peso en el resultado final, individualmente es algo sumamente importante.

La riqueza de cada nación sin duda alguna radica en base en la libertad, pero si un país a pesar de que tenga libertad en otros aspectos, si en su gobierno se sufre de corrupción, opresión y abuso de la fuerza, todo lo demás en realidad no sirve, pues indica que la libertad es solo ejercida para unos cuantos.

Exigir mayor libertad económica, salvaguardad derechos y libertades individuales es sin duda lo primordial para llegar a ser una nación más prospera, pues se nota al investigar cada país que aparece dentro de los primeros lugares del ranking, se sufre la falta de ello debido a la corrupción, opresión y uso de la fuerza, se necesita una nación que deje fuera a los corruptos, que dejen ser libre al mercado.

Pero es importante cambiar la mentalidad respecto a instituciones y gobierno, se pueden abrir grandes oportunidades, si optamos por un modelo económico que respete el mercado y que deje que se ajuste por si solo y se pone al emprendedor como agente fundamental dentro de la economía, es muy probable que exista mayor prosperidad y crecimiento dentro de los países.
La planificación central no funciona, el socialismo no funciona, cada que el gobierno persigue sus objetivos con políticas económicas, los resultados son lo contrario a lo que se plantea; más libertad significa más riqueza, incluso sin libertad de expresión, todo se convierte en expresión del gobierno

Es por todo lo anterior que debe reconocerse la eficiencia y el valor del mercado, del LIBRE MERCADO, pues cuando lo rompemos, surgen otro tipo de problemáticas como mercados negros, de desabastecimiento (como pobreza extrema), entre otros; para lograrlo es necesario primero el respeto a los derechos individuales: la vida, la propiedad privada y la libertad.

La mayoría de los argumentos contra el libre mercado se basan en la falta de confianza en la libertad misma.

-Milton Friedman

\section{REFERENCIAS:}

Boaz, D. (2007). Las raíces del liberalismo. El Cato, 1-14.

Cardoso, H. A. (2006). El origen del neoliberalismo: tres perspectivas. Espacios Públicos, vol. 9, núm. 18, 2006, pp. 176-193.

Casar, M. A. (1996). Las bases políticoinstitucionales del poder presidencial en México. Política y Gobierno, volumen III, número 1, 1er semestre de 1996, pp 61-92

Chang, H.-J. (2019). Conferencia magistral "Desarrollo Productivo para la Igualdad y el Crecimiento. Senado de la república. Ciudad de México, México.

https://www.canaldelcongreso.gob.m $\mathrm{xl}$ 
Contreras, G. B. (1987). La libertad económica: Elemento de un nuevo orden político- económico. Política. Revista de Ciencia Política, (13), 5976.

Daron Acemoglu y James A. Robinson. (2012). Por qué fracasan los países. España, Barcelona: Ediciones Deusto.

Diccionario de la lengua española (2001) Real Academia Española.

Fernández, P. M. (2003). De lo político a la política. Liberalismo, el otro límite de la legitimidad. Memoría presentada para optar al grado.

Hayek, F. A. (1960). "Los fundamentos de la libertad. Estados Unidos : University of Chicago Press.

Hervey, D. (2007). Breve historia del neoliberalismo. España: Ediciones Akal.

Isaac Katz. (2019) El Economista, Periódico El Economista. Recuperado el 2020 de

https://www.eleconomista.com.mx/op inion/Libertad-economica-201705150034.html

Karlsson, S. (21 de Enero de 2005). Mises Institute. Obtenido de El fracaso del índice de libertad económica: https://mises.org/es/library/elfracaso-del-\%C3\%ADndice-delibertad- econ\%C3\%B3mica

Llamas, A., \& Rodrigo de León, G. (2005). El poder ejecutivo de los Estados Unidos Mexicanos. Nostra Ediciones. Pp 21-25

Mariña, A., y Cámara Izquierdo, S. (2019). Movimientos internacionales de capital y la inserción Neoliberal de México en el mercado mundial. En C. N. Bustamante, America Latina: movimientos de capitales y su efecto sobre el modelo liderado por las exportaciones (págs. 87-121). Ciudad de México.

Mises, L. V. (2007). EL socialismo; análisis económico y sociológico. España, Universidad Francisco Marroquín: Unión Editorial.

Palley, T. (2011). The Rise and Fall of Exportled Growth. The Levy Economics Institute Working Paper,2-24., Recuperado de http://www.levyinstitute.org/pubs/wp_ $\underline{675 . p d f}$.

PLACER, J. L. (6 de Octubre de 2018). Diario de León. Obtenido de ¿Para qué sirve la libertad económica?: https://www.diariodeleon.es/articulo/t ribunas/sirve-libertadeconomica/20180610040001177354 2.html

Ramírez Villalobos, E. (Mayo-junio 2012). El desarrollo del capitalismo en México en la segunda mitad del siglo XIX. Economía Informa núm. 374, 27.

Siero, A. L. (11 de Enero de 2016). Gestiopolis. Obtenido de Capitalismo, ideología y globalización: https://www.gestiopolis.com/capitalis mo-ideologia-globalizacion/

The Heritage Foundation. (2020). Obtenido de 2020 Index of Economic Freedom: https://www.heritage.org/index/countr y/mexicoc

Vázquez, I. (19 de Setiembre de 2016º). elcato.org. Obtenido de LoS beneficios de la libertad económica: https://www.elcato.org/los-beneficiosde-la-libertad-economica 